\title{
Avaliação de fatores primários e secundários no desenvolvimento da cárie dentária em pacientes infantis: um estudo piloto
}

Evaluation of primary and secondary factors in the development of dental caries in infant patients: a pilot study Evaluación de factores primarios y secundarios en el desarrollo de caries dental en pacientes infantiles: un estudio piloto

Laura Imbriani BENTO

Departamento de Odontologia, Universidade de Ribeirão Preto, UNAERP, 14096-900 Ribeirão Preto - SP, Brasil Yara Teresinha Correa Silva SOUSA Departamento de Odontologia, Universidade de Ribeirão Preto, UNAERP, 14096-900 Ribeirão Preto - SP, Brasil https://orcid.org/0000-0002-7671-1656

Francyenne Maira Castro GONÇALVES Departamento de Odontologia Preventiva e Restauradora, Faculdade de Odontologia, UNESP Univ. Estadual Paulista, 16015-050 Araçatuba - SP, Brasil https://orcid.org/0000-0003-4102-1484 Nayara Gonçalves EMERENCIANO Departamento de Odontologia Preventiva e Restauradora, Faculdade de Odontologia, UNESP Univ. Estadual Paulista, 16015-050 Araçatuba - SP, Brasil https://orcid.org/0000-0003-3614-3129

Marcelle DANELON

Departamento de Odontologia Preventiva e Restauradora, Faculdade de Odontologia, UNESP Univ. Estadual Paulista, 16015-050 Araçatuba - SP, Brasi

Departamento de Odontologia, Universidade de Ribeirão Preto, UNAERP, 14096-900 Ribeirão Preto - SP, Brasil https://orcid.org/0000-0003-2091-649X

\section{Resumo}

A prática de alimentação, além de fatores secundários, recebe destaque na etiologia da Cárie na Primeira Infância (CPI). Essa doença é definida como a presença de uma ou mais superfícies dentárias cariadas, perdidas ou obturadas em crianças com idade inferior a 6 anos, tendo o presente estudo como objetivo avaliar as práticas alimentares na infância, bem como fatores secundários, e sua relação com a cárie dentária de pacientes atendidos na Clínica de Odontopediatria I e II da Universidade de Ribeirão Preto. Inicialmente, este estudo foi submetido ao Comitê de Ética em Pesquisa da UNAERP, o qual foi aprovado, e posteriormente, foi realizada a coleta de dados. Para a avalição, foi aplicado um questionário estruturado abordando alguns itens sobre hábitos da criança e seu responsável: Alimentação; Escovação; Erupção dentária; Dieta; Avaliação comportamental; Presença de lesões cariosas; entre outros. Após os dados serem tabulados e analisados de forma descritiva, foi possível evidenciar que das 34 crianças participantes do estudo, $97 \%$ realizaram amamentação natural (peito). A alimentação industrializada iniciou-se em 53\% dos participantes entre 1 e 2 anos; $59 \%$ escovavam os dentes 3x/dia ou mais, sendo que 35\% não recebiam auxílio. $97 \%$ utilizavam pasta de dente fluoretada. A maioria (65\%) relatou não usar o fio dental; 53\% tiveram o nascimento do primeiro dente antes dos 6 meses de idade e $38 \%$ entre 6 meses e 1 ano. $59 \%$ consomem doces $1 \times 1$ dia, sendo que $79 \%$ nunca foram ao nutricionista. Ao exame clínico, $24 \%$ apresentaram lesão de mancha branca (75\% ativa). É possível concluir que hábitos nocivos à saúde bucal de crianças pré-escolares podem resultar em consequências negativas para os elementos dentários.

Descritores: Cárie Dentária; Dieta Cariogênica; Pré-Escolar.

\section{Abstract}

In addition to secondary factors, the practice of feeding is highlighted in the etiology of caries in early childhood (CPI). This disease is defined as the presence of one or more carious tooth surfaces, lost or obtained in children under 6 years of age, with the present study as an objective to evaluate eating practices in childhood, as well as secondary factors, and their relationship with the dental caries of patients seen at the Pediatric Dentistry Clinic I and II of the University of Ribeirão Preto. Initially, this study was submitted to the Research Ethics Committee of UNAERP, which was approved, and subsequently, data collection was performed. For the evaluation, a structured questionnaire was applied addressing some items on children's habits and their guardians: Nutrition; Brushing; Dental eruption; Diet; Behavioral evaluation; Presence of carious lesions; among others. After the data were tabulated and analyzed in a descriptive way, it was possible to evidence that out of the 34 children participating in the study, $97 \%$ performed natural (breast) breastfeeding. The industrialized feeding started in $53 \%$ of the participants between 1 and 2 years; $59 \%$ brushed their teeth $3 \times /$ day or more, and $35 \%$ did not receive any help. $97 \%$ used fluoridated toothpaste. Most (65\%) reported not using dental floss; $53 \%$ had their first tooth born before 6 months of age and $38 \%$ between 6 months and 1 year. $59 \%$ consumed candy $1 \times$ day, and $79 \%$ never went to the nutritionist. At clinical examination, $24 \%$ had white spot lesion $(75 \%$ active). It is possible to conclude that habits harmful to the oral health of preschool children can result in negative consequences for the dental elements.

Descriptors: Dental Caries; Diet, Cariogenic; Child, Preschool.

\section{Resumen}

Además de los factores secundarios, la práctica de la alimentación se destaca en la etiología de la caries en la primera infancia (IPC). Esta enfermedad se define como la presencia de una o más superficies dentales cariadas, perdidas u obtenidas en niños menores de 6 años, siendo el presente estudio un objetivo para evaluar las prácticas de alimentación en la infancia, así como los factores secundarios, y su relación con las caries dentales de los pacientes atendidos en la Clínica de Odontología Pediátrica I y II de la Universidad de Ribeirão Preto. Inicialmente, este estudio se presentó al Comité de Ética de la Investigación de la UNAERP, que fue aprobado, y posteriormente se realizó la recopilación de datos. Para la evaluación, se aplicó un cuestionario estructurado que abordaba algunos puntos sobre los hábitos de los niños y sus tutores: Nutrición; Cepillado; Erupción dental; Dieta; Evaluación del comportamiento; Presencia de lesiones cariadas; entre otros. Después de que los datos se tabularon y analizaron de manera descriptiva, fue posible evidenciar que de los 34 niños que participaron en el estudio, el $97 \%$ realizaba lactancia natural (de pecho). La alimentación industrializada comenzó en el 53\% de los participantes entre 1 y 2 años; el 59\% se cepilló los dientes 3 veces al día o más, y el $35 \%$ no recibió ninguna ayuda. El $97 \%$ usó pasta dental fluorada. La mayoría (65\%) informó no usar hilo dental; el $53 \%$ tuvo su primer diente antes de los 6 meses de edad y el 38\% entre los 6 meses y 1 año. El 59\% consumió caramelos 1 vez al día, y el $79 \%$ nunca fue al nutricionista. En el examen clínico, el $24 \%$ tenía una lesión de manchas blancas (75\% activas). Se puede concluir que los hábitos perjudiciales para la salud bucal de los niños en edad preescolar pueden tener consecuencias negativas para los elementos dentales.

Descriptores: Caries Dental; Dieta Cariógena; Preescolar.

INTRODUÇÃO

A cárie dentária é uma doença

bacteriana de origem multifatorial que se

estabelece caso o indivíduo apresente uma higiene bucal deficiente, dieta e microbiota cariogênicas, fatores socioeconômicos e ambientais desfavoráveis, tempo, $\mathrm{pH}$ alterado e um hospedeiro susceptível ${ }^{1}$. 
Quando a cárie surge na fase préescolar (até 6 anos de idade), a American Academy of Pediatric Dentistry a considera como Early Childhood Caries, sendo classificada pela Associação Internacional de Odontopediatria (IAPD) como Cárie na Primeira Infância (CPI), segundo a Declaração de Bangkok, desde 2019. A mesma caracteriza-se pela presença de um ou mais dentes decíduos cariados (com ou sem cavidade), restaurados/obturados ou perdidos ${ }^{2}$. Os fatores etiológicos de maior relevância são: higiene bucal deficiente, devido ao desconhecimento das crianças e seus responsáveis acerca da técnica correta de escovação e à falta de auxílio durante a higienização bucal, bem como uma dieta com alto índice de açúcar, muito comum nesta faixa etária. Ainda há fatores secundários predisponentes, como: peso, temperamento da criança, baixa escolaridade e renda familiar. Clinicamente, a CPI afeta com maior frequência os incisivos superiores, sendo a progressão da lesão cariosa classificada em 3 estágios: Estágio 1 (leve), onde as faces vestibular e palatina são afetadas, mas sem acometer a borda incisal dos dentes; Estágio 2 (moderado), onde as faces afetadas também são a vestibular e a palatina, mas com acometimento da borda incisal dos dentes; e Estágio 3 (severo), onde já há grande perda da estrutura dentária.

Existem dois protocolos de atendimento frente à CPI: preventivo, que se baseia na higienização da cavidade bucal da criança com dentifrícios fluoretados com no mínimo 1000 ppm $F$, além de manutenção de dieta com controle de açúcar, bem como curativo/reabilitador que consiste em tratamentos de choque para reverter o risco, diminuindo a microbiota cariogênica e aumentando a resistência do dente, com aplicações tópicas de flúor, selantes ou até mesmo procedimentos restauradores/reabilitadores quando necessário ${ }^{3}$.

Este trabalho teve como proposição avaliar as práticas alimentares na infância, bem como fatores secundários, e sua relação com a cárie dentária de pacientes atendidos na Clínica de Odontopediatria I e II da Universidade de Ribeirão Preto-UNAERP.

MATERIAL E MÉTODO

Este estudo foi inicialmente submetido e aprovado pelo Comitê de Ética em Pesquisa (CEP-Plataforma Brasil, CAAE: 25854519.6.0000.5498) da Universidade de Ribeirão Preto-UNAERP. A seguir, responsáveis $(n=40)$ por crianças atendidas nas Clínicas de Odontopediatria I e II da Universidade de
Ribeirão Preto-UNAERP foram convidados a participarem do estudo, bem como as respectivas crianças. Dessa amostra inicial $(n=40), 34$ aceitaram dar continuidade ao estudo. Adicionalmente, um Termo de Consentimento Livre e Esclarecido (TCLE) foi assinado pelos responsáveis das crianças.

A amostra foi composta efetivamente por 34 crianças atendidas regularmente nas Clínicas de Odontopediatria I e II da Universidade de Ribeirão Preto-UNAERP, de ambos os gêneros, com idade de 3-12 anos. As crianças que participaram dos atendimentos clínicos foram encaminhadas pelo Sistema Único de Saúde (SUS), e receberam tanto tratamento preventivo quanto curativo, dependendo da situação clínica. Os responsáveis pelas crianças em atendimento foram convidados a participar desse estudo. Os dados coletados foram os seguintes: exame clínico intra-bucal para avaliar a condição bucal da criança (Lesão de mancha branca e seu status; Dentes restaurados; Número de lesões de cárie; Tratamento endodôntico) e questionário abordando alguns itens sobre hábitos e rotina da criança. O questionário foi dividido em duas partes, sendo que na primeira foram coletados os dados da criança em relação a hábitos de higiene oral e dieta; a segunda parte abordou informações clínicas da cavidade bucal da criança. O formulário foi desenvolvido para ser aplicado especificamente neste estudo, e previamente à sua aplicação definitiva, foi prétestado para adequação das perguntas aos objetivos do estudo. Os dados foram tabulados e analisados de forma descritiva, levando-se em consideração as variáveis de estudo, que são os itens abordados no questionário.

RESULTADOS

As Figuras 1 e 2 mostram, respectivamente, os dados referentes à amamentação natural (peito) e o seu período de duração. Foi possível observar que $97 \%$ dos participantes realizavam amamentação natural, tendo esta prática uma duração, na grande maioria (55\%), acima de 1 ano de vida.

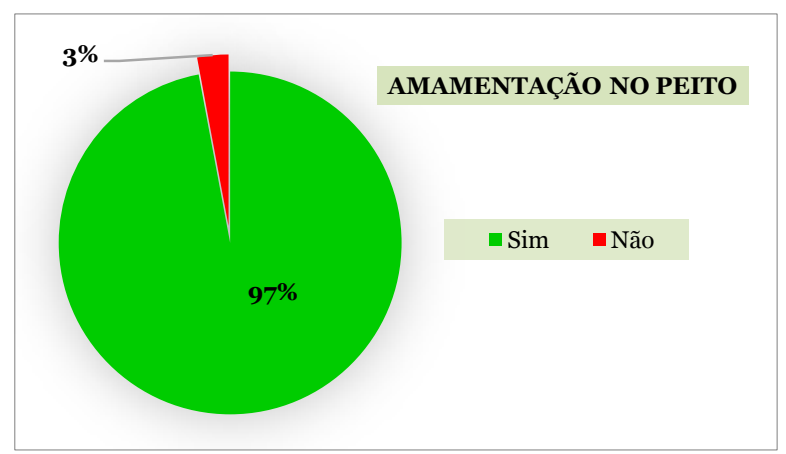

Figura 1: Amamentação no peito $(n=34)$. 


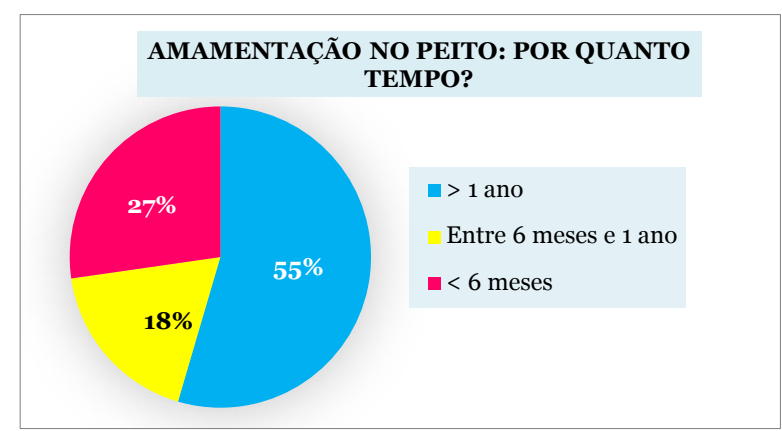

Figura 2: Duração da amamentação no peito $(n=34)$.

A Figura 3 mostra a relação da introdução de mamadeira. Na Figuras 4 e 5 é possível observar, respectivamente, o momento da introdução da alimentação natural e industrializada, de acordo com a idade da criança. Na maioria (56\%), a mamadeira foi introduzida abaixo de 1 ano de vida. $29 \%$ dos participantes não utilizaram mamadeira. Em relação à alimentação natural, 94\% das crianças do estudo foram introduzidas com idade inferior a 1 ano de vida, $3 \%$ entre 1 e 2 anos de vida e $3 \%$ acima dos 2 anos de vida. Para os dados de alimentação industrializada, foi possível observar que $53 \%$ consumiam entre 1 e 2 anos de vida.

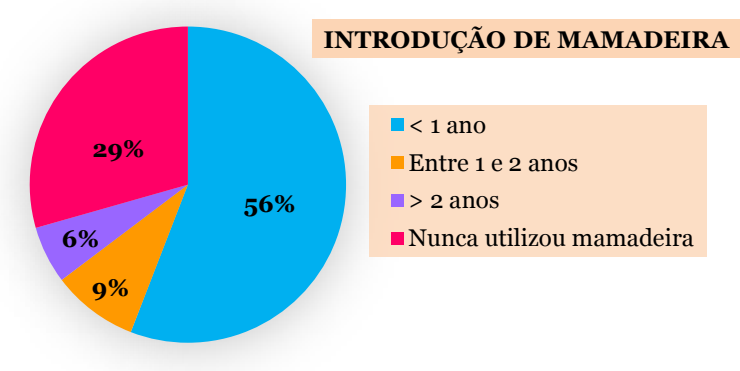

Figura 3: Distribuição da introdução da mamadeira $(n=34)$.

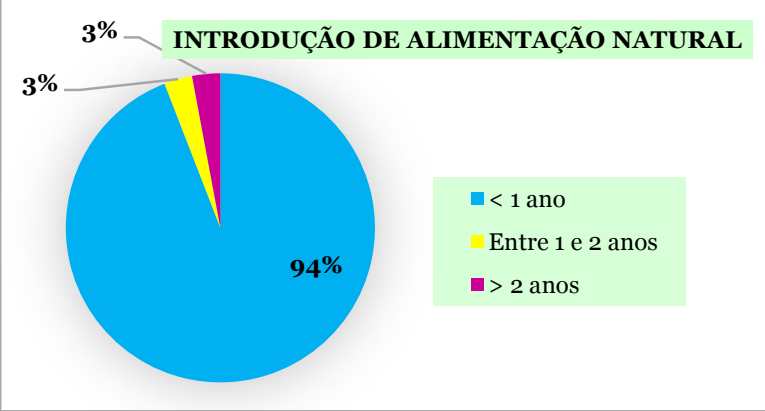

Figura 4: Introdução da alimentação natural $(n=34)$.

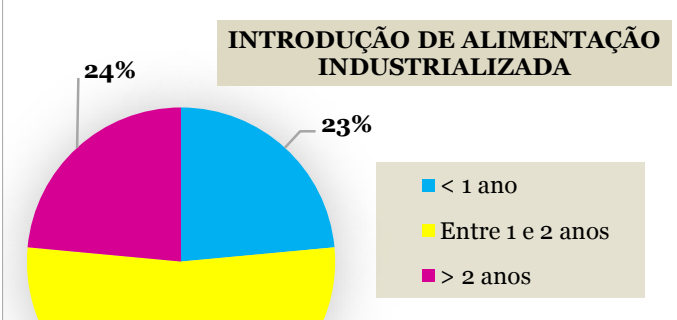

$53 \%$

Figura 5: Introdução da alimentação industrializada $(n=34)$.
A Figura 6 relaciona o número de escovações diárias em relação ao auxílio durante as mesmas por parte dos responsáveis. É possível evidenciar que $59 \%$ das crianças realizam essa prática mais que $3 x /$ dia, sendo que $35 \%$ não recebem auxílio durante a escovação e apenas $24 \%$ recebem. Já $33 \%$ das crianças realizam a escovação $2 x /$ dia, entretanto $21 \%$ a fazem sem auxílio. Apenas $9 \%$ responderam que a higienização é realizada $1 \times /$ dia, sendo que $6 \%$ também não recebem auxílio.

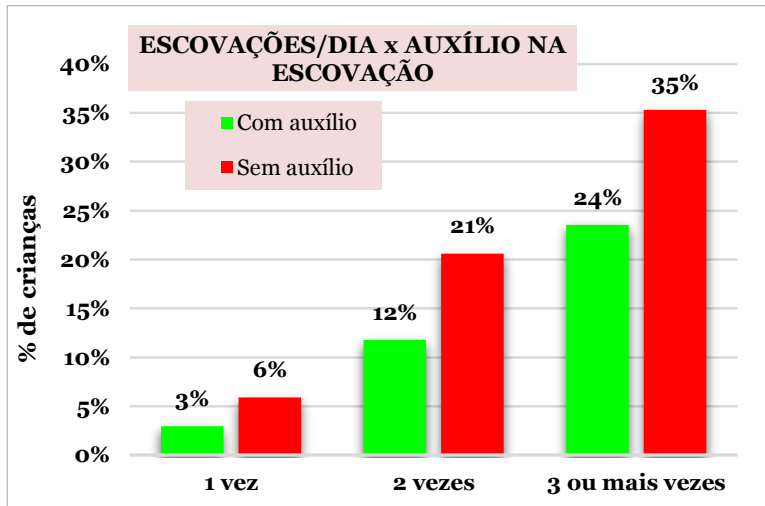

Figura 6: Distribuição das escovações/dia em relação ao recebimento de auxílio na escovação $(n=34)$.

A Figura 7 relaciona 0 número de escovações realizadas por dia com a quantidade de lesões de cárie presentes na cavidade bucal. É possível evidenciar que $41 \%$ das crianças que escovam os dentes 3 vezes ou mais por dia são as que apresentaram o maior índice de lesões cariosas (mais que 1 lesão de cárie) no momento do exame clínico.

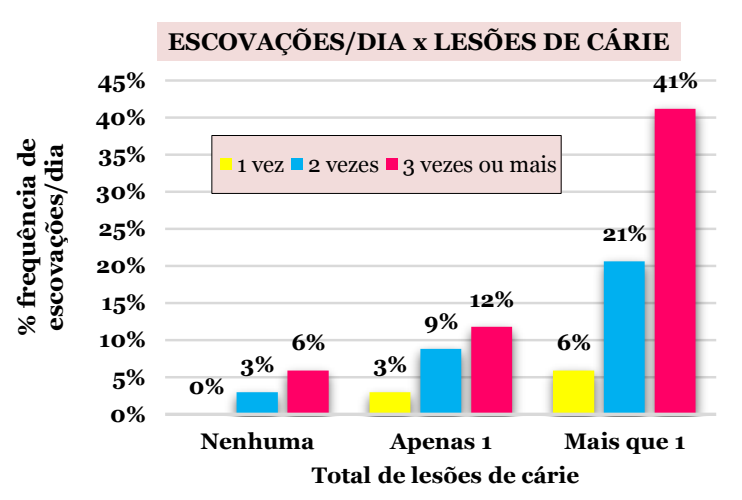

Figura 7: Distribuição das escovações/dia em relação às lesões de cárie $(n=34)$.

As Figuras 8 e 9 mostram, respectivamente, a relação da utilização do dentifrício ("pasta de dente") fluoretado ou não, bem como do fio dental. Das 34 crianças (100\%) que utilizam dentifrício, 97\% relataram que era fluoretada, e apenas $3 \%$ sem flúor. $\mathrm{Na}$ análise sobre a utilização do fio dental como rotina de higiene bucal, $65 \%$ dos participantes relataram o não uso, e apenas 35\% relataram que utilizam durante a escovação. 


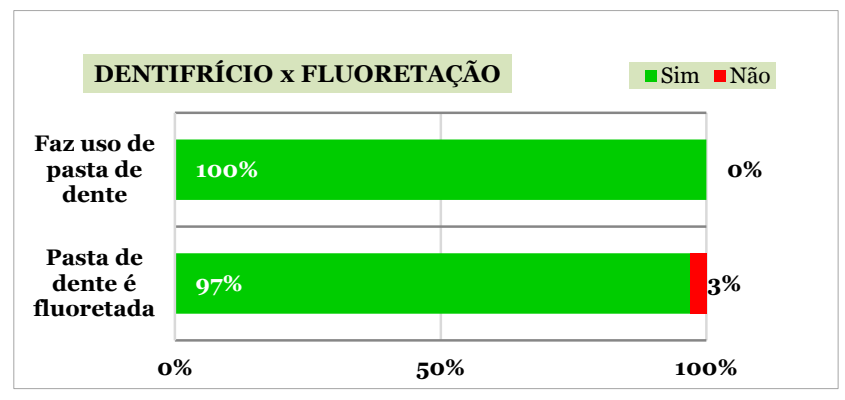

Figura 7: Avaliação do uso de dentifrício ("pasta de dente") com ou sem fluoretação $(n=34)$.

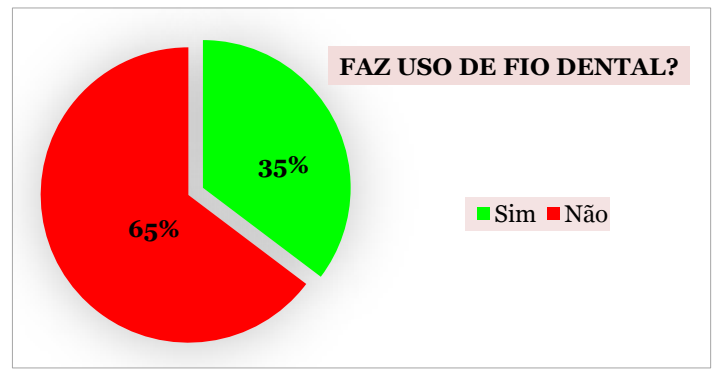

Figura 8: Avaliação do uso de fio dental $(n=34)$.

As Figura 9, 10, 11 e 12 avaliam, respectivamente, a época de nascimento do primeiro dente, introdução do dentifrício ("pasta de dente") fluoretado, assim como a ocorrência da aplicação tópica de flúor profissional e o local de aplicação (Clínica particular ou Universidade). Dos 34 entrevistados, 53\% tiveram erupção do primeiro dente entre 6 meses e 1 ano de idade, sendo que em $38 \%$ a erupção ocorreu abaixo de 6 meses, e apenas $9 \%$ acima de 1 ano. A pasta de dente fluoretada foi introduzida em $43 \%$ dos participantes acima de 2 anos de idade, 39\% entre 1 e 2 anos e $18 \%$ abaixo de 1 ano. Com intuito de verificar o acompanhamento profissional, foi questionado se o paciente realizou aplicação tópica de flúor, onde $85 \%$ relataram que sim, sendo esta efetuada em Universidade (76\%).

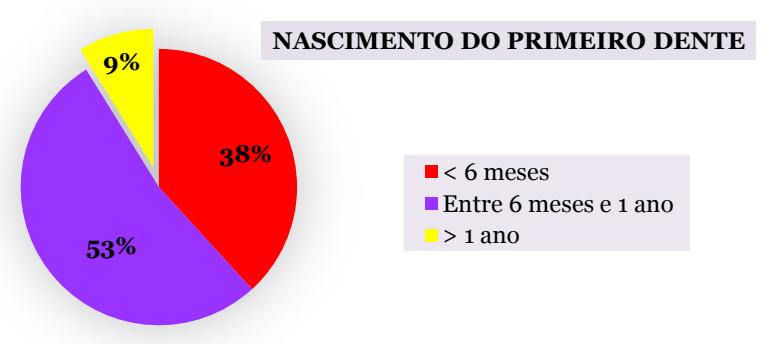

Figura 9: Avaliação do nascimento do primeiro dente $(\mathrm{N}=34)$.

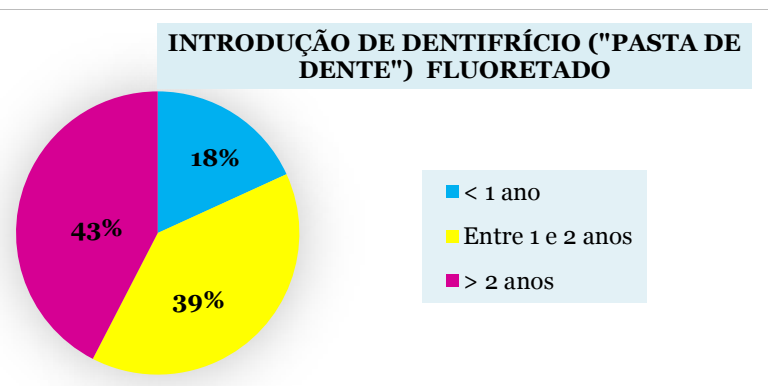

Figura 10: Introdução do dentifrício fluoretado $(\mathrm{N}=34)$.

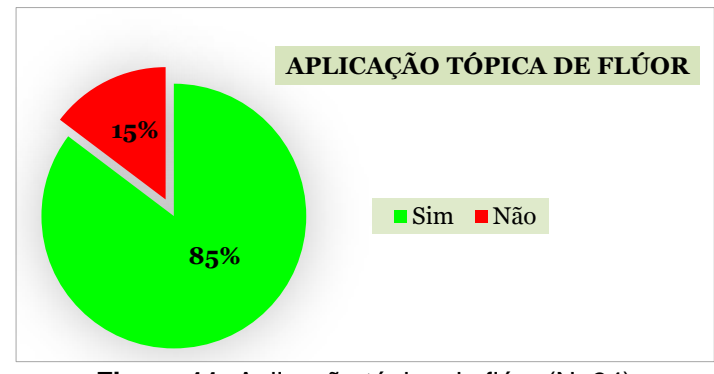

Figura 11: Aplicação tópica de flúor ( $\mathrm{N}=34)$.

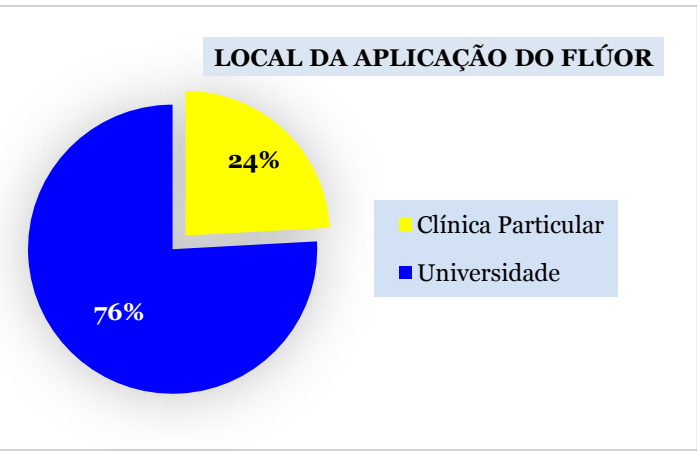

Figura 12: Local da aplicação tópica de flúor $(\mathrm{N}=34)$.

A Figura 13 mostra os dados da frequência de consumo de doces. As Figuras 14 e 15 apresentam, respectivamente, os referentes ao uso de antibióticos e multivitamínicos. Na Figura 16 é possível observar o acesso à consulta ao nutricionista, enquanto a Figura 17 apresenta o percentual das crianças praticantes de atividade física.

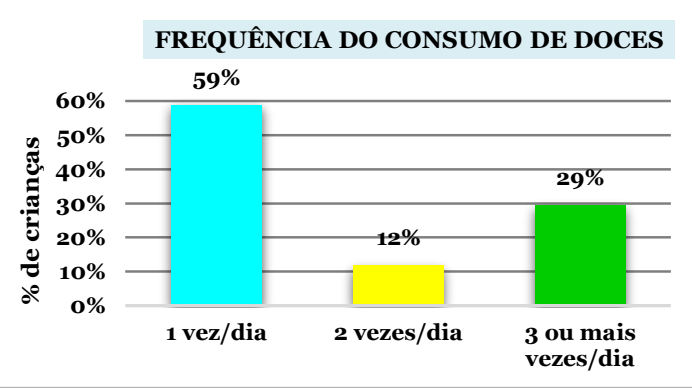

Figura 13: Avaliação da frequência de consumo de doces $(\mathrm{N}=34)$

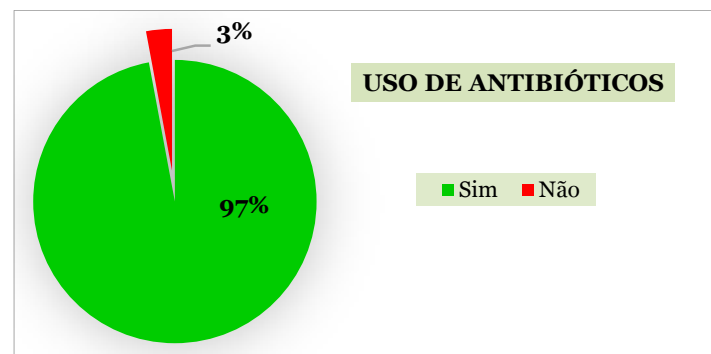

Figura 14: Avaliação da frequência do uso de antibióticos ( $\mathrm{N}=34)$.

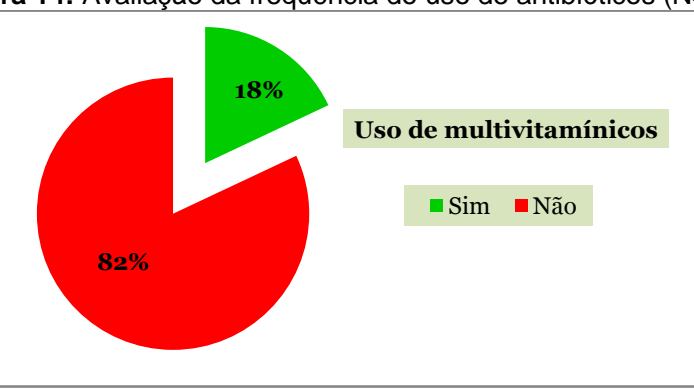

Figura 15: Avaliação da frequência do uso de multivitamínicos $(\mathrm{N}=34)$. 


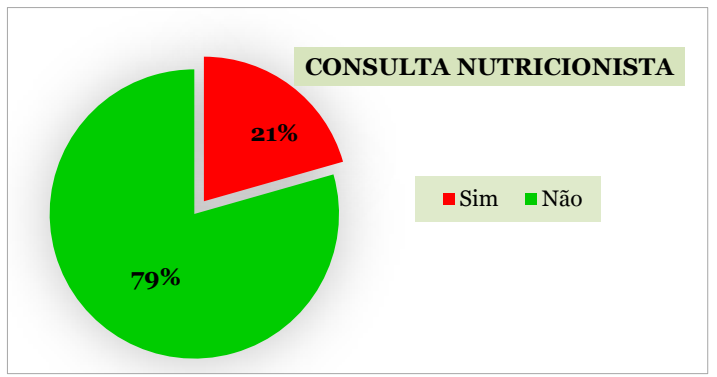

Figura 16: Consulta ao nutricionista ( $\mathrm{N}=34)$.

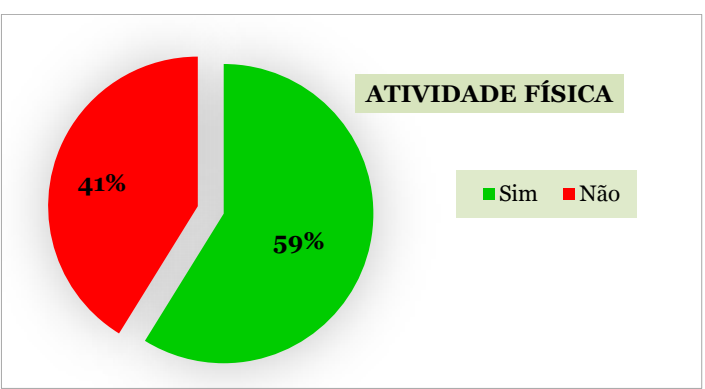

Figura 17: Prática de atividade física ( $\mathrm{N}=34)$.

Destaca-se que $59 \%$ dos participantes relataram que pelo menos $1 \mathrm{x} / \mathrm{dia}$ fazem ingestão de doce, $12 \%$ - 2x/dia e $29 \%$ - 3x/dia ou mais. A ingestão de antibiótico foi realizada pela maioria das crianças $(97 \%)$. Em relação ao consumo de suplemento vitamínico, 18\% relataram sua utilização. Sabendo-se da importância de alimentação saudável, foi questionado aos participantes se já buscaram auxílio profissional através de nutricionista para as crianças, evidenciando que apenas uma minoria de $21 \%$ já teve ou tem essa prática e $79 \%$ nunca a tiveram. Em relação à atividade física, foi possível observar que 59\% praticam e $41 \%$ relataram não a realizar.

Dados sobre a presença de lesão inicial de cárie (lesão de mancha branca) estão apresentados na Figura 18, enquanto seu status pode ser observado na Figura 19.

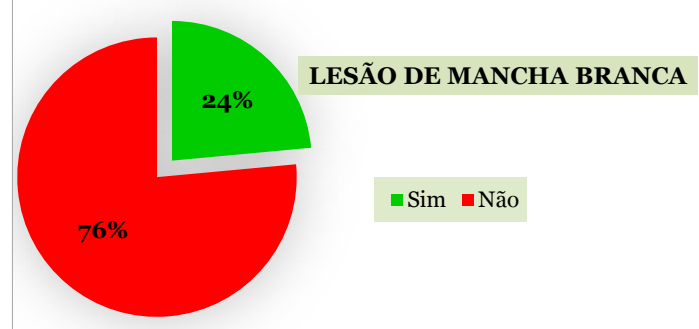

Figura 18: Avaliação da presença da lesão de mancha branca $(n=34)$.

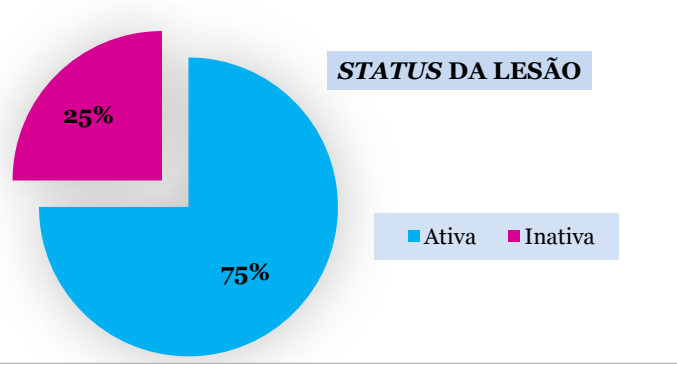

Figura 19: Status lesão de mancha branca $(n=34)$.
A análise mostrou que $76 \%$ dos pacientes não possuíam lesão de mancha branca, entretanto, dos $24 \%$ que apresentaram, $75 \%$ encontravam-se ativas.

O número de dentes restaurados observados durante o exame clínico está apresentado na Figura 19. As Figuras 20 e 21 exibem, respectivamente, o número de lesões de cárie e o número de dentes tratados endodonticamente. Foi possível verificar que $74 \%$ das crianças apresentavam mais que 1 dente restaurado e apenas $6 \%$ não possuíam dentes restaurados. Além disso, $68 \%$ apresentavam a cavidade bucal com mais de 1 lesão de cárie e $35 \%$ já haviam sido submetidas ao tratamento endodôntico.

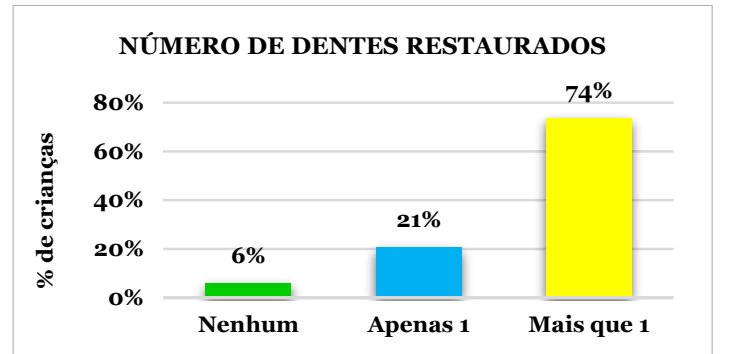

Figura 21: Avaliação da presença de dentes restaurados $(n=34)$.

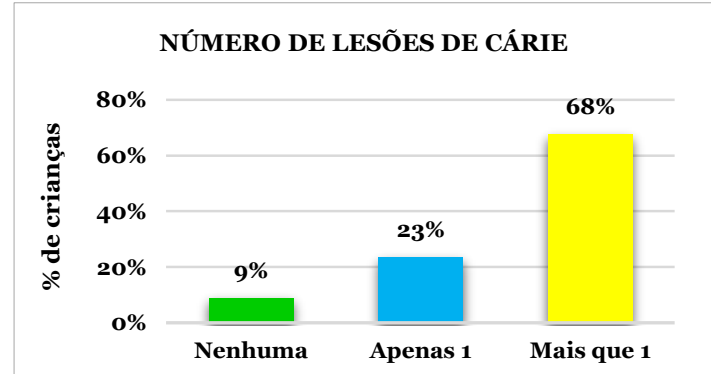

Figura 21: Avaliação da presença de lesões de cárie $(n=34)$.

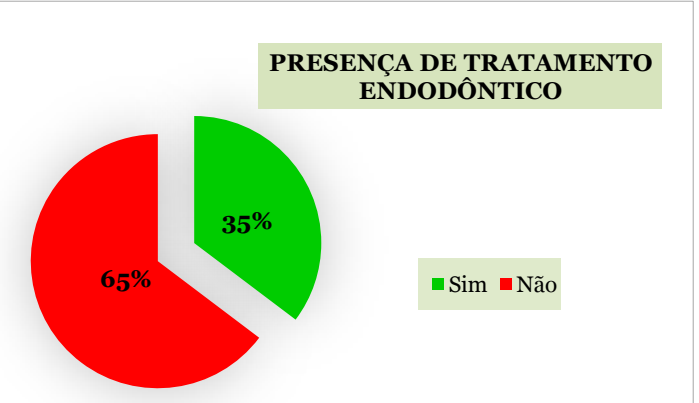

Figura 22: Avaliação da presença de: dentes tratados endodonticamente $(n=34)$.

DISCUSSÃO

A magnitude da prevenção da CPI é altamente discutida no meio Odontopediátrico, porém apesar da grande divulgação de métodos preventivos, a doença continua estabelecida como um problema de saúde pública ${ }^{4}$.

O aleitamento materno é uma prática fundamental de alimentação adequada para os bebês, fornecendo um aporte de calorias, nutrientes e água que os mesmos necessitam, 
contribuindo para 0 seu crescimento $e$ desenvolvimento. É possível destacar que $97 \%$ dos participantes deste estudo puderam realizar a amamentação natural (peito) - um bom índice, considerando-se a importância do leite materno. A Organização Mundial da Saúde (OMS) e a Sociedade Brasileira de Pediatria (SBP) recomendam que os bebês sejam amamentados com leite materno até os 2 anos ou mais, devendo ser exclusivo até o sexto mês de vida. Ao iniciar a introdução de novos alimentos é importante que a criança tenha acesso, inicialmente, a alimentos naturais, que garantam o suprimento adequado de nutrientes que ela necessite. Nota-se que $94 \%$ das crianças participantes deste estudo, segundo relatado pelos responsáveis, iniciaram a ingestão de alimentos naturais antes do primeiro ano de vida, $3 \%$ entre 1 e 2 anos e $3 \%$ após os 2 anos; porém, mostra-se preocupante que, $53 \%$ tiveram acesso a alimentos industrializados entre 1 e 2 anos e $23 \%$ antes do primeiro ano de vida, períodos muito precoces para a introdução dos mesmos. Deve-se retardar o consumo de alimentos, como bolachas, salgados, refrigerantes, doces, entre outros, pois a alimentação inadequada no primeiro ano de vida pode desencadear efeitos permanentes na saúde e bem-estar da criança, afetando seu crescimento e metabolismo, podendo acarretar, futuramente, diabetes, hipertensão, doenças cardiovasculares e obesidade, assim como ser um fator pré-disponente para a CPI.

Observa-se que $59 \%$ das crianças realizam a escovação dentária 3 ou mais vezes ao dia, sendo que destas, 35\% não recebem auxílio durante a escovação e apenas 24\% recebem. Cascaes et al. ${ }^{6}$ recomendam que a escovação dos dentes de crianças menores de seis anos de idade deve ser realizada ou acompanhada por um adulto, visto que ainda estão em desenvolvendo de sua destreza manual e aspecto cognitivo. Este auxílio por parte dos responsáveis deve ser priorizado até que as crianças obtenham habilidade suficiente que Ihes permita realizar uma higienização independente de forma satisfatória. A Figura 7, que relaciona escovações por dia $x$ lesões de cárie, evidencia que dos $59 \%$ dos participantes que escovam os dentes 3 ou mais vezes ao dia, $41 \%$ apresentaram mais que uma lesão de cárie. Portanto, esses dados nos permitem evidenciar que somente a quantidade de escovações por dia sem destreza e preparação manual para remoção total do biofilme torna a higiene ineficiente, e consequentemente, os elementos dentários continuam propícios à cárie da mesma forma.
Outro fator relevante avaliado neste estudo foi a utilização de pasta de dente fluoretada pelos participantes. A escovação regular com dentifrício fluoretado é a principal intervenção não profissional para prevenir a cárie, contudo o efeito preventivo varia de acordo com as diferentes concentrações de flúor na pasta de dente, com concentrações mais elevadas (acima de 1000 ppm F) associadas ao aumento do controle da cárie ${ }^{7}$. No tocante às diretrizes da utilização de dentifrícios fluoretados, a AAPD ${ }^{3}$ incentiva que a escovação dos dentes seja realizada com pastas fluoretadas pelo menos duas vezes ao dia, após a erupção dentária, sendo estas também na concentração acima de 1100 ppm F. A resposta dos entrevistados foi unânime quanto à utilização diária de dentifrícios, sendo que $97 \%$ relataram que a pasta de dente que empregam é fluoretada.

Nesse mesmo sentido, uma revisão sistemática publicada por Walsh et al. ${ }^{7}$ relatou a importância do uso de pasta de dente fluoretada de maneira racional, uma vez que formulações com maior concentração de flúor aumentam o risco de fluorose dentária. Sendo assim, esse cuidado deve ser considerado e ensinado aos responsáveis para evitar que tal patologia ocorra na dentição permanente. No presente estudo, apenas $18 \%$ relataram que iniciaram o uso de pasta de dente fluoretada antes de um ano de idade, sendo um importante fator a ser considerado, pois $53 \%$ dos respondentes tiveram o nascimento do primeiro dente antes dos 6 meses de idade, e $38 \%$ entre 6 meses e 1 ano. Em geral, crianças que não fazem uso de fluoretos são aquelas abaixo dos 3 anos de idade, sendo a justificativa de seus responsáveis para a não utilização dos mesmos baseada em evitar a ingestão excessiva de flúor ou a falta de informação sobre a necessidade do uso de pastas fluoretadas desde o nascimento do primeiro dente decíduo, por volta dos 6 a 8 meses de idade.

Ainda é possível destacar que $65 \%$ das crianças avaliadas nunca fizeram ou não fazem uso de fio dental diariamente. Sabe-se que este mecanismo de limpeza é um importante complemento durante a higienização dos dentes, sendo indispensável para o controle do biofilme e a prevenção de cárie na região interproximal. Worthington et al. $^{8}$ avaliaram a eficácia de dispositivos de limpeza interdentais utilizados em casa associados à escovação, em comparação com apenas a escovação, para prevenir e controlar doenças periodontais, cárie e placa bacteriana, concluindo que a associação durante a higienização torna ainda mais capaz a 
redução de gengivite do que se for realizada apenas a escovação. Ainda é imprescindível que os responsáveis estejam presentes no momento da escovação da criança, principalmente das mais jovens, até que obtenham técnica e destreza manual necessárias para efetuaram uma higienização eficiente e completa sozinhas.

Em relação à frequência do consumo de doces, pode-se observar que $59 \%$ das crianças consomem alimentos açucarados fielmente ao menos uma vez por dia, sendo que $79 \%$ dos responsáveis relataram que nunca levaram seus filhos ao nutricionista. Sabe-se que é significativo 0 acompanhamento de um profissional para orientar corretamente as etapas de introdução alimentar, bem como os alimentos ideais que sejam adequados para as necessidades de cada faixa etária da criança. Dada a importância do aconselhamento nutricional, este índice mostra-se preocupante pelo fato dos responsáveis nunca terem considerado a relevância de modificar e adequar a alimentação da criança com o auxílio de um profissional. A American Dietetic Association adverte que a alimentação e a nutrição têm influência direta na progressão da $\mathrm{CPI}$, o que valida a importância de uma nutrição equilibrada para a saúde bucal. Uma dieta inadequada e pouco diversificada, pode, ainda, afetar a oferta de energia e densidade proteica das crianças, desencadeando uma carência de micronutrientes. Segundo Vaz et al. ${ }^{9}$, o uso de suplementos multivitamínicos mostra-se como uma opção favorável e eficaz, sendo habilitados para suprir os déficits nutricionais da alimentação infantil. Porém, é preocupante que $82 \%$ dos participantes entrevistados nunca lançaram mão do uso de suplementos vitamínicos (Figura 15). É necessário que este tópico seja abordado com mais frequência em programas preventivos e educacionais, instruindo os responsáveis acerca da suplementação na infância.

Quanto às atividades físicas, os responsáveis se mostraram conscientes de sua importância para a saúde e bem-estar da criança, trazendo benefícios não só físicos, mas também psicológicos e até sociais. $59 \%$ dos participantes afirmaram praticar atividades esportivas extracurriculares ou no âmbito escolar, semanalmente (Figura 17). Se por um lado a prática de atividade física pode desencadear inúmeros benefícios, sua ausência também pode ser prejudicial: o sedentarismo é capaz de representar inúmeros riscos para a saúde, bem como, quando associado à uma dieta inadequada, ser um fator predisponente para a CPI. Também é possível relacionar o estabelecimento da CPI ao uso de antibióticos, visto que, além de alguns possuírem a presença de açúcares (sacarose) em sua composição, outros medicamentos ainda contêm alta acidez, o que favorece a desmineralização da estrutura dentária. Neste estudo, 97\% dos participantes relataram já terem feito uso de antibióticos ao menos uma vez na vida (Figura 14). Segundo Xavier et al. ${ }^{10}$ é relevante destacar que apenas a ingestão isolada dos medicamentos não é capaz de desencadear a doença, porém a utilização de um antibiótico reflete, indiretamente, que a criança se encontra em uma situação de saúde debilitada, e consequentemente, outras condições como alimentação e hábitos de higiene, geralmente também se mostram alteradas.

Durante a realização do exame clínico, $76 \%$ dos pacientes não apresentaram lesões de mancha branca, enquanto $24 \%$ apresentaram. É relevante que, desses $24 \%, 75 \%$ se mostraram com status ativo (Figura 19). A mancha branca está relacionada com a desmineralização do esmalte, que, quando diagnosticado em sua fase inicial, apresenta-se apenas com estrutura parcialmente desmineralizada, sendo passível de remineralização. Sendo essas manchas ativas precursoras das lesões cavitadas de cárie, é necessário que sejam diagnosticadas corretamente e controladas para evitar o desenvolvimento e estabelecimento da doença. ${ }^{11}$ As abordagens de tratamento frente a essas lesões baseiam-se em técnicas de microabrasão no esmalte com pasta de pedra pomes e ácido fosfórico, aplicação de verniz, instrução e auxílio de higiene oral às crianças, com indicações de pastas de dente fluoretadas. CONCLUSÃO

Analisados os dados obtidos e
compreendidos os tópicos que levaram à
discussão desse tema, é possível concluir que o conjunto de hábitos nocivos à saúde bucal de crianças pré-escolares, como os exemplificados neste estudo, pode resultar em consequências negativas para os elementos dentários das mesmas, podendo, em alguns casos, influenciar a saúde dos futuros dentes permanentes. Desse modo, ressalta-se a importância de programas educativos para que a prevenção da Cárie na Primeira Infância seja realizada o mais breve possível, com instruções às crianças e seus respectivos responsáveis acerca da higiene oral, alimentação, além de hábitos que devam ser adequados e/ou corrigidos.

REFERÊNCIAS

1. Mangueira DFB, Passos IA, Pereira AMBC, 
Oliveira AFB. Cárie e erosão dentária: uma breve revisão. Odontol Clin Cient. 2011;10: 121-24.

2. Alhabdan YA, Albeshr AG, Yenugadhati N, Jradi $H$. Prevalence of dental caries and associated factors among primary school children: a population-based cross-sectional study in Riyadh, Saudi Arabia. Environ Health and Prev Med. 2018;23:60.

3. American Academy of Pediatric Dentistry. Policy on Use of Fluoride. AAPD. 20192020;55-6.

4. Seow WK. Early Childhood Caries. Pediatr Clin North Am. 2018;65:941-54.

5. Mazo-Tomé PLD, Suárez-Rodríguez $M$. Prevalence of Exclusive Breastfeeding in the Healthy Newborn. Bol Med Hosp Infant Mex. 2018;75:49-56.

6. Cascaes AM, Peres KG, Peres MA, Demarco FF, Santos I, Matijasevich A, et al. Validade do padrão de higiene bucal de crianças aos cinco anos de idade relatado pelas mães. Ver Saúde Pública. 2011;45:668-75.

7. Walsh T, Worthington HV, Glenny AM, Marinho VC, Jeroncic A. Fluoride toothpastes of different concentrations for preventing dental caries. Cochrane Database Syst Rev. 2019; 3(3): CD007868..

8. Worthington HV, MacDonald L, Pericic TP, Sambunjack $D$, Johnson TM, Imai $P$ et al. Home use of interdental cleaning devices, in addition to toothbrushing, for preventing and controlling periodontal diseases and dental caries. Cochrane Database Syst Rev. 2019; 4(4):CD012018.

9. Vaz MA, Oliveira GG, Pinheiro MS, Medeiros EFF. Suplementação na infância e a prevenção da carência de micronutrientes: Artigo de revisão. Rev Med Saúde Brasília. 2017; 6(1):116-31.

10. Xavier AFC, Cavalcanti AL, Oliveira MC, Vieira FF. Antibióticos líquidos de uso pediátrico: caracterização físico-química. Rev HU. 2011;37:397-401.

11. Ancira-González L, Esparza-Villalpando V, Garrocho-Rangel A, Pozos-Guillén A. White Spot Lesion Remineralisation Agents in Primary Teeth: A Systematic Review. Oral Health Prev Dent. 2018;16:391-400.

\section{CONFLITO DE INTERESSES}

Os autores declaram não haver conflitos de interesse

\section{AUTOR PARA CORRESPONDÊNCIA}

\author{
Marcelle Danelon \\ Departamento de Odontologia, \\ Universidade de Ribeirão Preto, UNAERP, \\ 14096-900 Ribeirão Preto - SP, Brasil \\ Telefone: 16-36036781 \\ email: mdanelon@unaerp.br
}

